\title{
Évaluation et labellisation
}

\section{Evaluation and status recognition}

\section{G. Amarenco}

Reçu le 8 janvier 2010 ; accepté le 11 janvier 2010

(C) Springer-Verlag France 2010

Évaluation. Maître mot de notre pratique médicale actuelle. À côté de l'evidence-based medicine, il s'agit de la deuxième vraie révolution survenue, ces dernières années, dans notre manière de soigner mais aussi de gérer les patients et les structures.

Cette évaluation est avant tout médicale. Elle nous permet de prendre du recul et de moduler nos pratiques en se détachant de nos habitudes parfois bien ancrées, mais bien peu réfléchies... La poésie de la «tradition orale » a bien vécu, et cela est souvent bien bénéfique pour nos patients. Notre pratique professionnelle est désormais encadrée par ce type de réflexion. Pertinence des examens complémentaires, apport bénéfice-risque des stratégies thérapeutiques sont ainsi discutés, conduisant très vraisemblablement à une meilleure prise en charge.

Mais cette évaluation n'est pas que médicale, et si elle a été si expressément promue par les autorités de santé, c'est bien en raison de sa pertinence médicoéconomique : soigner mieux au moindre coût, optimiser les ressources médicales, humaines et logistiques, améliorer l'efficience des outils diagnostiques et thérapeutiques en fonction des contraintes budgétaires sont autant d'impératifs... bien peu masqués.

Nous n'avons plus guère la maîtrise de l'organisation, du contenu, des modalités d'application et du caractère obligatoire de cette évaluation. Les « autorités », peut-être à juste titre car comptables des deniers publics et partant de la rationalisation des soins, ont la mainmise sur tous ces éléments.
Pourtant, les sociétés savantes comme la SIFUD-PP doivent s'impliquer dans une telle démarche, ne serait-ce que pour devenir et rester des interlocuteurs privilégiés des tutelles. S'engager dans un tel processus est le meilleur moyen de garder la maîtrise médicale de tout ce qui concerne la sphère périnéale et particulièrement la neuro-urologie.

Pour cela, deux axes majeurs peuvent être individualisés.

La formation est capitale, car le socle de la connaissance est le lit et le ciment de pratiques médicales homogènes, réfléchies, réactualisées, enrichies d'expériences non seulement hexagonales mais internationales. La création, cette année, du DIU de neuro-urologie va dans ce sens (http://diu-neurourologie.jimdo.com/). Notre revue Pelvi-périnéologie doit rester le moyen de communication essentiel de cette néodiscipline, tant en ce qui concerne la formation continue que l'aspect recherche clinique. La pérennité de la revue qui passe, rappelons-le, par l'indexation Medline, est un atout important et un vrai enjeu.

Mais la labellisation des laboratoires d'urodynamique, l'individualisation des centres de références, la graduation des unités fonctionnelles en fonction de l'allocation en ressources médicales, paramédicales et de l'importance du plateau technique sont une autre démarche indispensable. C'est une ambition récente de la SIFUD-PP qui doit entrer désormais dans une phase active.

Ce challenge doit être relevé par tous, hospitaliers ou privés, universitaires ou non.

Conflit d'intérêt : aucun.

G. Amarenco $(\bowtie)$

e-mail : gerard_amarenco@yahoo.fr 